\title{
ERRATA, VOLUME 53
}

A. S. Galbraith and J. W. Green, $A$ note on the mean value of the Poisson kernel.

p. 316, display (5). For “ $m ! 2^{m}$ " read “ $m ! 2^{m+\rho}$."

W. H. Mills, $A$ prime-representing function.

p. 604, displays (3), (4), and (5). For " $3-n$ " read " $3^{-n}$." For " $3-n-1$ " read " $3^{-n-1}$."

S. Chandrasekhar, The transfer of radiation in stellar atmospheres.

p. 679 , line 2 from the bottom. For " $\sigma\left(\mu, \varphi ; \mu_{0}, \varphi_{0}\right) \mu$ " read " $\sigma\left(\mu, \varphi ; \mu_{0}, \varphi_{0}\right) / \mu . "$

T. R. Hollcroft, The April Meeting in New York.

p. 723, line 10. For "53-5-121" read "53-3-121."

H. J. Hamilton, Mertens' theorem and sequence transformations.

p. 786, line 15. Read " $\neq$ " for " $=$."

H. E. Salzer, An "empirical theorem" which is true for the first 618 cases, but fails in the 619th. Abstract 53-9-308.

p. 908 , line 4 of the abstract. For "618" read "6186."

p. 908, line 5 of the abstract. For "2006" read "20006." 